\title{
The Utility of Local Anesthesia for Neurosurgical Interventions in a Low-Resource Setting: A Case Series
}

\author{
Jessica Eaton ${ }^{1,2} \cdot$ Asma Bilal Hanif ${ }^{3}$ - Suzgisam Mzumara ${ }^{4}$ Anthony Charles An,5,6 $^{1,3,}$
}

Published online: 11 October 2017

\begin{abstract}
Introduction Trauma is a major contributor to global morbidity and mortality, and injury to the central nervous system is the most common cause of death in these patients. While the provision of surgical services is being recognized as essential to global public health efforts, specialty areas such as neurosurgery remain overlooked.

Method This is a retrospective case review of patients with operable lesions, such as extra-axial hematomas and unstable depressed skull fractures that underwent neurosurgical interventions under local anesthesia.

Results A total of 13 patients underwent neurosurgical intervention under local anesthesia. Two and three patients with burr hole decompression of epidural and subdural hematomas, respectively; seven patients had elevation of depressed skull fractures and lastly one patient had an aspiration of a brain abscess. All patients survived with and without residual neurological deficits.

Conclusion Access to resources and staff required to deliver general anesthesia is challenging in resource-poor settings. We have therefore begun performing emergent interventions under local anesthesia, with or without conscious sedation. While some patients had some minor residual weakness after the procedure, the degree of neurological deficit was improved from that observed before the procedure in all patients.
\end{abstract}

Anthony Charles

anthchar@med.unc.edu

UNC -Project Malawi, Lilongwe, Malawi

2 University of Louisville School of Medicine, Louisville, KY, USA

3 Department of Surgery, Kamuzu Central Hospital, Lilongwe, Malawi

4 Department of Radiology, Kamuzu Central Hospital, Lilongwe, Malawi

5 Department of Surgery, University of North Carolina, Chapel Hill, NC, USA

6 UNC School of Medicine, Gillings School of Global Public Health, University of North Carolina, 4008 Burnett Womack Building, CB 7228, Chapel Hill, NC, USA

\section{Introduction}

Globally, trauma remains one of the leading causes of death and disability [1], particularly in low- and middleincome countries where incidence and mortality of trauma remain disproportionately high [2]. Brain and spinal cord injuries remain the most common cause of mortality in trauma patients [3]. In order to attenuate trauma-related morbidity and mortality, it is imperative that we optimize care for patients with traumatic brain injury (TBI).

In recent years, the delivery of surgical care has been recognized as an essential element of global public health [4], with particular emphasis in general surgical, obstetric, and orthopedic care. Despite the high burden and serious consequences of TBI, the delivery of basic neurosurgical care has largely remained unaddressed [5]. The list of essential procedures set forth in Disease Control Priorities 
includes only one neurosurgical procedure: burr hole evacuation of subdural hematoma. This is discordant with the relative prevalence of TBI, estimated at $150-170$ per 100,000 in sub-Saharan Africa [6], which can be significantly improved with neurosurgical interventions when patients have extra-axial hematomas, depressed skull fractures, or associated brain abscesses.

There are an estimated 1.0-3.1 operating theaters per 100,000 people in sub-Saharan Africa, as compared to 14.3 and 25.1 theaters per 100,000 people in the USA and Europe, respectively [7]. In resource-poor settings, the diminished operating theater capacity, shortage of nursing and anesthesia providers, coupled with the lack of expertise in neurosurgical procedures and the perceived poor prognosis of patients with TBI have resulted in the lack of prioritization of operative neurosurgical care delivery in favor of general surgical and orthopedic procedures. In our environment, it is both pragmatic and necessary to be able to provide emergent neurosurgical interventions to patients in the absence of a specialized team and outside the operating theater.

Malawi is a landlocked country in southeast Africa with a population if 16.4 million and a GDP of $\$ 1100$ per capita [8]. It currently has the designation of the poorest country in the world and is hence representative of issues related to surgical care delivery in low- and middle-income countries (LMICs), such as low per capita healthcare spending, and an inadequate nursing, anesthetic, and surgical workforce resulting in limited access to surgical care.

Kamuzu Central Hospital is a 1000-bed tertiary care center with five operating theaters, serving a catchment of 6 million people in the capital of Malawi. The surgical department is staffed by consultants in general surgery, orthopedic surgery, urology, pediatric surgery, and neurosurgery. There is also a general surgical residency. There are four beds available in a high-dependency unit, with 1-to-1 nursing coverage and monitoring, as well as five beds available in an intensive care unit equipped with mechanical ventilation. X-rays, ultrasound, and CT scanning, as well as basic laboratory investigations, are available. The availability of both radiology and laboratory studies is not constant, due to resource limitations and staffing issues. Anesthesia services at $\mathrm{KCH}$ are provided by non-physician clinical officers akin to physician assistants. Clinical officers undergo a shortened medical training of about 18 months, with most of the training clinically based. These clinical officers also manage the ICU care of patients. Most acute situations, including intubation and other airway management, in the emergency department and the inpatient units are managed by general surgery residents and attendings who have all undergone Advanced Trauma Life Support (ATLS) training.
In order to overcome both infrastructural- and personnel-related impediment to neurosurgical operating room access, we began performing emergency neurosurgical operative interventions at the bedside under local anesthesia, with or without conscious sedation. We herein present our case series of 13 neurosurgical operative interventions and describe four representative cases (Table 1).

\section{Case presentations}

In our setting, prior to any operative intervention under local anesthesia, radiologic localization of underlying neurological lesion is mandatory for perioperative planning and preparation to improve chances of success. Operative intervention was limited to the following diagnoses: epidural and subdural hematomas, depressed skull fractures, and intracerebral abscesses.

\section{Epidural and subdural hematoma evacuation}

After obtaining consent from patient or next of kin as appropriate, the patients were sedated with $100 \mathrm{mg}$ intravenous meperidine and $10 \mathrm{mg}$ intravenous diazepam and given $1 \mathrm{~g}$ of ceftriaxone. The head was shaved, and the region of the procedure was washed with both alcohol and $0.5 \%$ chlorhexidine. About $1 \%$ lidocaine was infiltrated into the skin, subcutaneous fat, and galea in the region of the intended burr hole. Patients received intravenous lactated Ringer's fluid throughout the procedure and the postoperative period.

Using sterile technique, a scalpel was used to make a 1.5-in. cut to the level of the bone, and a periosteal elevator was used to strip the periosteum. A Hudson-Brace hand drill with a perforator bit was then used to drill a burr hole over the region of the hematoma. A rongeur was used to expand the burr hole up to $2 \mathrm{~cm}$ if necessary, and the blood was carefully evacuated using suction and forceps. For subdural hematomas, a cruciate incision in the dura mater overlying the hematoma was made. Intravenous tubing was inserted gently into the burr hole to wash the entire area of the hematoma with normal saline. A subgaleal drain was left in place and removed after $24 \mathrm{~h}$. Postoperatively, patients received analgesia and phenobarbital as seizure prophylaxis. After discharge, patients were seen in neurosurgical clinic every week for 1 month. At the time of clinic review, all patients were either in the same condition as discharge, or slightly improved as described in each case. We present two and three patients with extradural and subdural hematoma, respectively, following head injury.

PK was a 59-year-old man who presented to $\mathrm{KCH}$ after being assaulted with a machete. On admission, vital signs 
Table 1 Patient characteristics and neurological outcomes

\begin{tabular}{lllll}
\hline Patient & Sex & Age (years) & Procedure & Outcome at discharge \\
\hline TC & M & 33 & Epidural hematoma evacuation & Residual left hemiparesis \\
PK & M & 59 & Epidural hematoma evacuation & No deficits \\
LF & M & 39 & Subdural hematoma evacuation & No deficits \\
RC & M & 75 & Subdural hematoma evacuation & Residual moderate ataxia \\
SZ & M & 50 & Subdural hematoma evacuation & Residual right hemiparesis \\
JN & M & 16 & Elevation of depressed skull fracture & No deficits \\
AY & M & 35 & Elevation of depressed skull fracture & Residual right hemiparesis \\
LK & M & 15 & Elevation of depressed skull fracture & Residual right hemiparesis \\
PM & F & 8 & Elevation of depressed skull fracture & No deficits \\
PT & M & 8 & Elevation of depressed skull fracture & Minor residual full-body weakness \\
EB & F & 1 & Elevation of depressed skull fracture & No deficits \\
OY & M & 12 & Elevation of depressed skull fracture & No deficits \\
CD & M & 22 & Brain abscess aspiration & No deficits \\
\hline
\end{tabular}

were within normal limits. Examinations revealed an initial GCS of 14, with only slight confusion, and equal and reactive pupils. The associated frontoparietal scalp and right eyelid laceration were sutured. A skull radiograph revealed a minimally depressed fracture of the frontal bone at the site of the scalp laceration. Subsequently, a head CT scan (Fig. 1) revealed a depressed comminuted right frontal bone fracture with underlying epidural hematoma and pneumocephalus, without significant brain edema. An

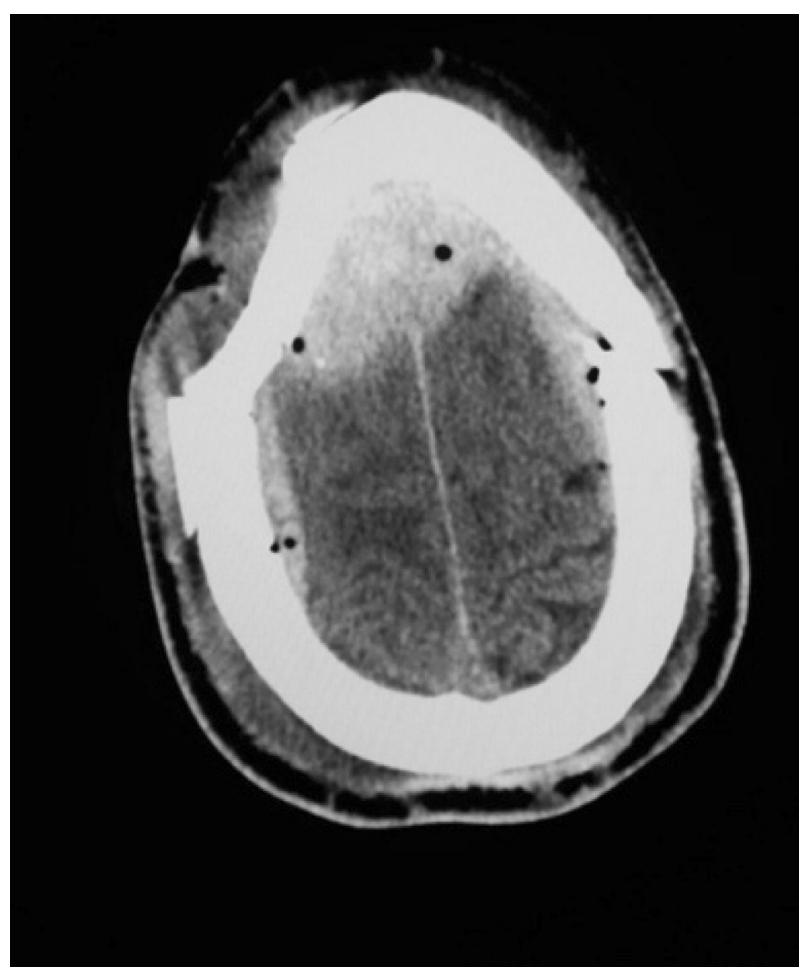

Fig. 1 Depressed comminuted right frontal bone fracture with underlying frontal and bilateral parietal epidural hematoma and pneumocephaly operating theater was not immediately available, so the patient was taken to the surgical HDU, where the wound was reopened and the epidural hematoma was evacuated through the existing bone defect. The fracture was subsequently elevated using leverage from a small hemostat. Postoperatively, the patient received a full course of antibiotics. He was discharged from the high-dependency unit the following day and discharged from the hospital on 5 days later with a GCS of 15 and no neurological deficits.

$\mathrm{RC}$ was a 75-year-old man who presented to $\mathrm{KCH}$ following a road-traffic crash. Unfortunately, he had been admitted to the medical department for management of his associated severe hypertension. At the time of his first examination by the surgical department, he was found to have a GCS of 8 , with an eye score of 2, a verbal score of 2 , and a motor score of 4 . Both pupils were dilated and sluggishly reactive to light. A CT scan done at the time of transfer (Fig. 2) showed a left subdural hematoma with obliteration of the left lateral ventricle and significant midline shift. A burr hole was done the same day, and the subdural hematoma was evacuated. The patient's GCS improved to 15 within $48 \mathrm{~h}$ and was discharged several weeks later via the medical department after continued management of his hypertension. At the time of discharge, the patient had moderate ataxia, but no other neurological deficits.

\section{Elevation of depressed skull fractures}

Elevation of depressed skull fractures was performed with the same general pre- and postoperative procedures described above with the exception of children under 5 years of age who received only a dose of intravenous meperidine without diazepam. All patients had associated 


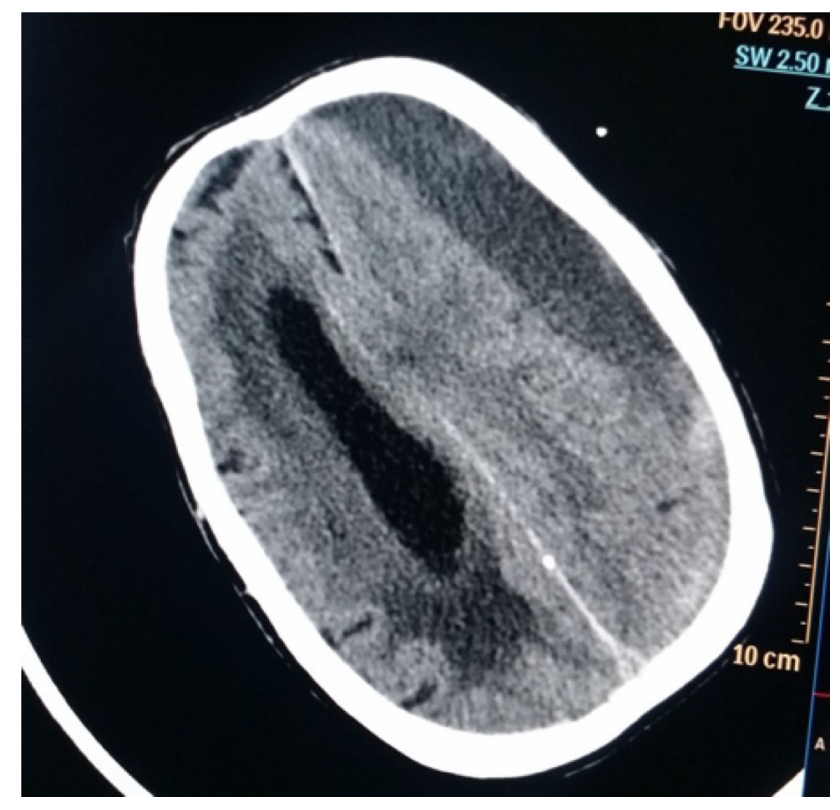

Fig. 2 Left subdural hematoma with obliteration of the left lateral ventricle and significant midline shift

scalp lacerations overlying the fractures, which were extended as necessary to access the skull fragments. After visualizing the fracture, any loose fragments, especially those protruding into the brain parenchyma, were removed. The leverage of a small hemostat was used to carefully realign the bones as much as possible. In one case (PM), we used a periosteal flap to close the dural defect. We then closed the scalp in a single layer. We present cases of seven patients who required elevation of depressed skull fractures and one patient who required elevation as well as abscess aspiration.

AY was a 35-year-old man who presented with a history of assault. He had previously been seen in the casualty department was treated as an outpatient. He returned due to decreased consciousness, episodes of convulsions, and right-sided hemiplegia. At the time of admission, his GCS was 10 with an eye of 4 , a verbal of 2 , and a motor of 4 . His pupils were slightly dilated but symmetric and reactive to light. He underwent $\mathrm{CT}$ scanning on the day of admission (Fig. 3) and was found to have a comminuted depressed skull fracture. Upon review after surgery, the patient's GCS was 8 , and he was intubated and admitted to the ICU. He underwent physiotherapy while in the hospital and was discharged 1 month after his admission. At that time, power on the right side had improved to 3/5. In follow-up clinic 1 week later, his weakness continued to improve.

CD was a 22-year-old man who was transferred from a district hospital with a history of being assaulted with an ax 8 days earlier. At admission, his vitals were normal, and he had no other injuries. His GCS was 15, and his pupillary

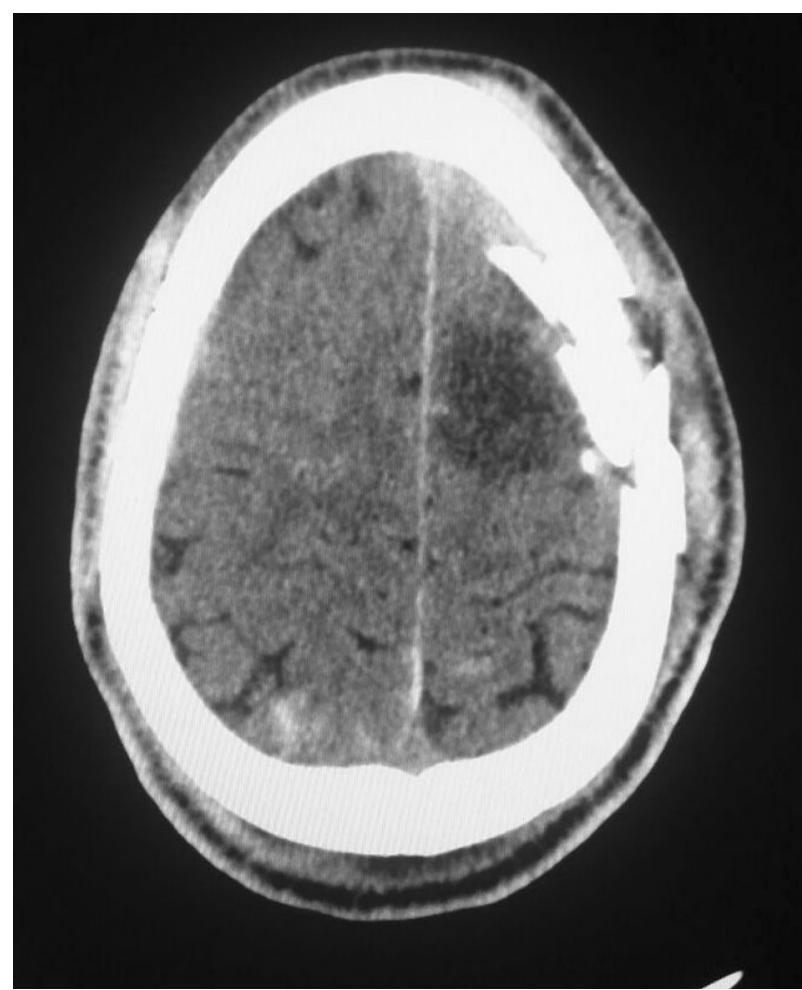

Fig. 3 Comminuted depressed skull fracture and underlying contusion in the dorsal portion of the left frontal lobe (region of the motor strip)

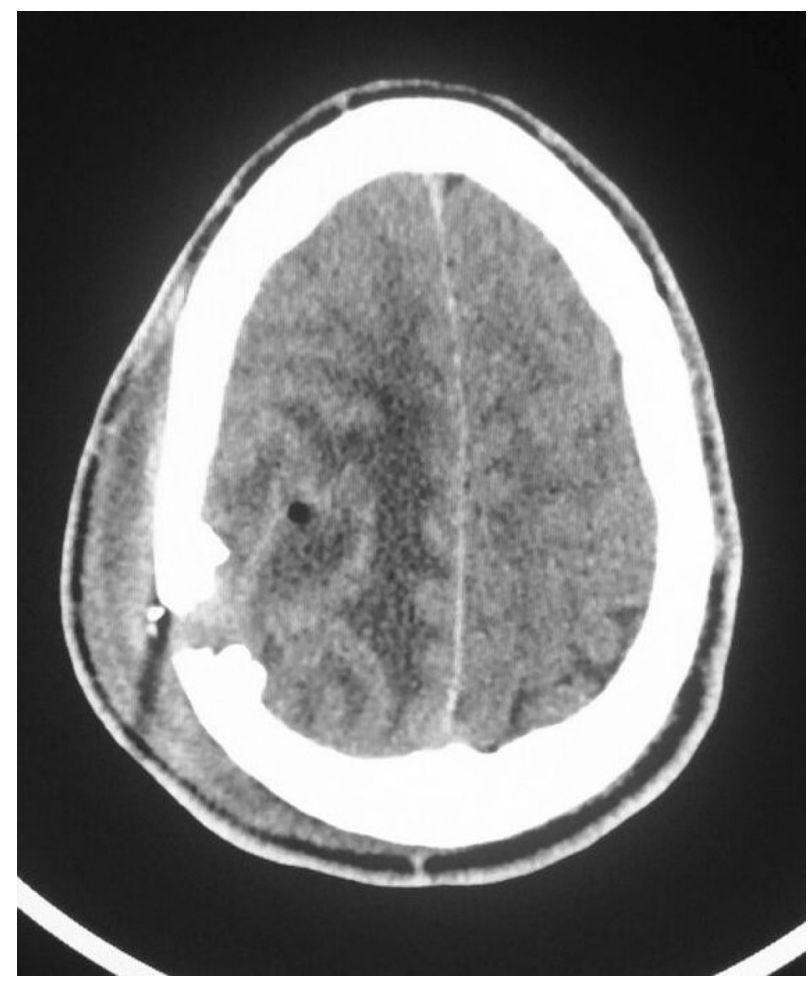

Fig. 4 Right parietal sub-galeal swelling with a comminuted skull fracture, and underlying abscess 
examination was normal, but he had an obvious right parietooccipital fracture. He was admitted to the general ward and underwent CT scan (Fig. 4) on hospital day 1, which showed subgaleal swelling, a comminuted skull fracture, and an underlying abscess. It was determined that the abscess should be accessible through the existing bone defect. The patient was sedated and received local anesthesia, and the existing wound was widened. A sterile cannula tip was inserted through the existing bone defect in the trajectory of the abscess, and a syringe was used to aspirate pus. About $10 \mathrm{ml}$ of pus was drained from the wound, which was then sutured. The patient was discharged from the hospital 5 days after admission with no neurological deficits.

\section{Discussion}

There is a significant burden of neurosurgical disease due to trauma that remains largely unaddressed in LMICs. In our setting, we have begun performing neurosurgical interventions for trauma patients at the bedside, under local anesthesia with or without conscious sedation, when general anesthesia and operating room access are unavailable. We have had favorable outcomes, as demonstrated by the above cases.

Globally, injury to the central nervous system is known to be the most common cause of mortality in trauma patients [3]. Likewise, in our setting, head injury is the most significant driver of mortality following trauma [9]. Patients with TBI are often assumed to inevitably have poor outcomes, and as a result of this bias, might not always receive the best possible care. It is critical that we recognize the importance of neurosurgical interventions in specific clinical scenarios following trauma, as corrective surgical intervention of lesions such as extradural hematomas result in favorable functional outcomes. Furthermore, the techniques do not require the physicians to secure an operating room, a nursing staff, or anesthesia services, all of which can be a challenge in our hospital.

It has been established that in patients with severe TBI, not all damage occurs at the time of injury, with secondary brain damage occurring in the hours and days following the initial insult [10]. Early implementation of the indicated management and interventions can have a significant impact on patient outcomes. In particular, when patients have extra-axial hematomas, evacuation should be performed within $4 \mathrm{~h}$ of the initial injury in order to improve the patient's chance at a favorable functional outcome $[11,12]$.

Local anesthesia and monitored sedation have long been used in the field of neurosurgery, beginning with Harvey Cushing, who operated almost solely under regional anesthesia, because of its lack of perioperative complications from inhalational anesthesia and the absence of pain receptors in the brain [13]. It became even more of an asset with increased understanding of cerebral localization. Currently, in high-income settings, patients undergoing cranial surgery are now often kept awake in order to describe sensations that come with stimulation of certain cortical areas [14]. Anesthetic technique has continued to be improved for awake surgery [15], and patients report comfort with the experience [16].

The addition of a sedative is a helpful adjunct while operating under local anesthesia, since the experience can be distressing to the patient. Because all anesthesia care providers at $\mathrm{KCH}$ are clinical officers rather than physicians, sedative dosing was generally done at the direction of the consultant neurosurgeon, with drug dosing based on ideal body weight. Importantly, we avoided the use of any sedative in children. The main complication of these drugs is respiratory depression [17]. For this reason, we ensure that all patients had a Guedel airway as appropriate and supplemental oxygen in addition to the availability of suction equipment. To avoid additional drug doses and subsequent risk of further respiratory depression, it is important that all patients receive preoperative CT scans and that the surgeons spend time planning the procedure beforehand in order to limit operative time. We also use an invasive, or non-invasive but continuous, blood pressure recording to monitor for unexpected hypertensive peaks that could cause bleeding during and after the procedure. If patients appeared to be too deeply sedated or the protection of their airway appeared to be at risk, they would be emergently intubated and transferred to the ICU. We have not seen any sedation-related complications in our setting so far. However, the expansion and development of comprehensive critical care services would inevitably benefit this patient population.

There are several opportunities for improvement demonstrated in our cases. All patients with residual neurological deficits were treated in a delayed fashion. Some patients with TBI were initially discharged from the emergency department only to return to the hospital with neurological deficits. This points to the need for review of the completeness of the trauma workup. These patients had lesions that would have been detected on CT scan, had it been performed on initial presentation. A careful history and thorough neurological examination must be performed, with care to follow-up on any evidence of TBI.

An additional challenge to the attenuation of TBI-related morbidity and mortality is posed by the shortage of neurosurgeons in sub-Saharan Africa, with an estimated 1 neurosurgeon per 9 million people in the southeastern Africa [18]. However, recent innovations are aimed at addressing the undersized workforce through twinning 
programs [19], and the training of general surgeons to perform basic and emergent neurosurgical procedures is underway [20].

\section{Conclusion}

Resource-poor settings face numerous challenges in providing lifesaving neurosurgical care to TBI patients. In our experience, several interventions including hematoma evacuation, depressed skull fracture elevation, and abscess aspiration can be safely and effectively performed under local anesthesia. We have demonstrated in our case series that these procedures can be performed safely. In all patients presented here, the neurological status of the patient improved from presentation, even if they did not completely return to their neurological baseline. Epidural and subdural hematomas can be rapidly fatal, and there is no substitute for corrective neurosurgical intervention. The techniques described here may be the only chance to save a patient's life. Furthermore, these procedures are simple and accessible for anyone with basic surgical skills. Teaching of this technique to general surgical providers in low-income settings may significantly reduce TBI-related morbidity and mortality.

Acknowledgements We are grateful for the support of the Departments of Surgery and Radiology at Kamuzu Central Hospital for their support of this work. We are particularly appreciative of the help of Webster Lungu for his help in obtaining CT images. This work was supported by the Fulbright Program and the National Institutes of Health Office of the Director, Fogarty International Center, Office of AIDS Research, National Cancer Center, National Heart, Blood, and Lung Institute, and the NIH Office of Research for Women's Health through the Fogarty Global Health Fellows Program Consortium comprised of the University of North Carolina, John Hopkins, Morehouse and Tulane (R25TW009340).

\section{Compliance with ethical standards}

Conflict of interest The authors declare that they have no conflict of interest.

\section{References}

1. Peden MM, McGee K, Krug E (eds) (2000) Injury: a leading cause of the global burden of disease, pp 1-51

2. de Ramirez SS, Hyder AA, Herbert HK, Stevens K (2012) Unintentional injuries: magnitude, prevention, and control. Annu Rev Public Health 33:175-191. doi:10.1146/annurev-publhealth031811-124558

3. Capone-Neto A, Rizoli SB (2009) Linking the chain of survival: trauma as a traditional role model for multisystem trauma and brain injury. Curr Opin Crit Care 15(4):290-294. doi:10.1097/ MCC.0b013e32832e383e

4. Meara JG, Hagander L, Leather AJM (2014) Surgery and global health: a Lancet Commission. Lancet 383(9911):12-13. doi:10. 1016/s0140-6736(13)62345-4

5. Mock CN, Donkor P, Gawande A, Jamison DT, Kruk ME, Debas HT (2015) Essential surgery: key messages from disease control priorities, 3rd edition. Lancet 385(9983):2209-2219. doi:10. 1016/s0140-6736(15)60091-5

6. Hyder AAWC, Puvanachandra P, Guruaj G, Kobusingye OC (2007) The impact of traumatic brain injuries: a global perspective. NeuroRehabilitation 22(5):341-353

7. Funk LMWT, Berry WR, Lipsitz SR, Merry AF, Enright AC, Wilson IH, Dziekan G, Gawande AA (2010) Global operating theatre distribution and pulse oximetry supply: an estimation from reported data. Lancet 376:1055-1061. doi:10.1016/S0140-

8. Agency CI. Malawi 2013 (cited 2017)

9. Tyson AF, Varela C, Cairns BA, Charles AG (2015) Hospital mortality following trauma: an analysis of a hospital-based injury surveillance registry in sub-Saharan Africa. J Surg Educ 72(4):e66-e72. doi:10.1016/j.jsurg.2014.09.010

10. Tang MELD (2009) Severe traumatic brain injury: maximizing outcomes. Mt Sinai J Med 76:119-128. doi:10.1002/msj.20106

11. Stone JLLR, Jonasson O, Baker RJ, Barrett J, Oldershaw JB (1986) Acute subdural hematoma: direct admission to a trauma center yields improved results. J Trauma 26(5):445-450

12. Seelig JMBD, Miller D, Greenberg RP, Ward JD, Choi SC (1981) Traumatic acute subdural hematoma: major mortality reduction in comatose patients treated with four hours. N Engl J Med 304(25):1511-1518

13. Bliss M (2005) Harvey cushing: a life in surgery. Oxford University Press, New York

14. Penfield W (1986) Combined regional and general anesthesia for craniotomy and cortical exploration. Part I. Neurosurgical considerations. Int Anesthesiol Clin 24(3):1-11

15. Bulsara KRJJ, Villavicencio AT (2005) Improvements in brain tumor surgery: the modern history of awake craniotomies. Neurosurg Focus 18(4):1-3

16. Danks RARM, Aglio LS, Gugino LD, Black PM (1998) Patient tolerance of craniotomy performed with the patient under local anesthesia and monitored conscious sedation. Neurosurgery $42(1): 28-36$

17. Surve RMBS, Reddy M, Philip M (2016) Use of dexmedetomidine along with local infiltration versus general anesthesia for burr hole and evacuation of chronic subdural hematoma. J Neurosurg Anestheiol 00:1-7

18. Saraceno BAJ, Prilipko L, Saxena S (2004) Atlas: country resources for neurological disorders. Academic Search Complete. http://www.who.int/mental_health/neurology/epidemiology/en/. Accessed 3 October 2017

19. Haglund MM, Kiryabwire J, Parker S, Zomorodi A, MacLeod D, Schroeder R, Muhumuza M, Merson M (2011) Surgical capacity building in Uganda through twinning, technology, and training camps. World J Surg 35(6):1175-1182. doi:10.1007/s00268-011$1080-0$

20. Coburger J, Leng LZ, Rubin DG, Mayaya G, Medel R, Ngayomela I, Ellegala D, Durieux ME, Nicholas J, Hartl R (2014) Multi-institutional neurosurgical training initiative at a tertiary referral center in Mwanza, Tanzania: where we are after 2 years. World Neurosurg 82(1-2):e1-e8. doi:10.1016/j.wneu.2012.09. 019 